\title{
Multidisciplinary Aesthetic Dental Treatment; Peg lateral with Congenitally Maxillary lateral Incisors
}

\author{
Hanali Abu Shilbayih*, Nezar Watted**, Muhamad Abu-Hussein \\ * Department Of Orthodontics And Pediatric Dentistry, Al-Quds University, Jerusalem, Palestine \\ ** University Hospital of Würzburg Clinics and Policlinics for Dental, Oral and Maxillofacial Diseases of the \\ Bavarian Julius-Maximilian- University Wuerzburg, Germany and Center for Dentistry research and \\ Aesthetics, Jatt, Israel
}

\begin{abstract}
Maxillary lateral incisors vary in form more than any other tooth in the mouth except the third molars. If the variation is too great, it is considered a developmental anomaly. Developmental alterations which are most commonly associated with maxillary lateral incisors either unilaterally or bilaterally are microdontia, hypodontia, dens invaginatus and dens evaginatus (talon cusp). Microdontia is a condition where the teeth are smaller than the normal size, which may involve all the teeth or be limited to a single tooth or a group of teeth. However, involvement of single tooth is a rather common condition, especially involving maxillary lateral incisor. Microdontia of maxillary lateral incisor is called as "peg lateral", that exhibit converging mesial and distal surfaces of crown forming a cone like shape. The root on such a tooth is usually shorter than usual. In this article, a case is reported of a young patient with one congenitally missing and one peg shaped lateral incisor. The patient was treated with a combination of orthodontics, periodontal surgery and aesthetic restorative dentistry interventions.
\end{abstract}

Keywords: Maxillary lateral incisors, Developmental anomaly, Peg lateral; Hypodontia,Dental implant, Aesthetics

\section{Introduction}

Microdontia is a term used to describe teeth that are smaller than normal. One of the most common forms of localized microdontia is that which affects the maxillary lateral incisors, called a "peg lateral". A peg shaped incisor has a marked reduction in diameter, extending from the cervical region to the incisal edge. $[1,2]$

The size of the teeth is predominantly genetically determined, depends on the race and can also be caused due to endocrinal disturbances. The prevalence in most studies varies from $0.8-8.4 \%$ of the population. $[2,3]$

The presence of maxillary peg shaped lateral incisors has been reported either on the left or right side of the jaw. Simultaneous presence of both the upper lateral incisors is a rare case. Simultaneous presence of both the upper lateral incisors is a rare case.[1,2,3,4]

The endocrine disturbances affect the size/form of the crown of teeth is not tenable unless such effects occur during morphodifferentiation, in utero or in the first year of life. Size and shape of the root, however, may be altered by disturbances in later periods. Disturbances in morphodifferentiation may affect the form and size of the tooth without impairing the function of the tooth and the function of ameloblast odontoblasts, thus the result may be a peg shaped/malformed tooth with enamel and dentine that may be normal in structure.[3,4,5]

Permanent tooth agenesis, maxillary lateral incisor microdontia, palatally displaced canines, and distoangulation of mandibular second premolars are frequently associated with maxillary lateral incisor agenesis, providing additional evidence of a genetic interrelationship in the causes of these dental anomalies.

Hypodontia, congenitally missing teeth is more common in permanent than primary dentition. A study described the prevalence of peg shaped laterals in the maxilla to be $7.5 \%$ in Asians and $1.6 \%$ in non-Asians. The occurrence being common in girls when compared to boys. [5]

The prevalence of hypodontia and congenital malformation in permanent teeth of Saudi Arabian male children was present in about 2.6-4\%; most frequently affected was the mandibular second premolars, maxillary laterals, and maxillary second premolars. Tooth malformations, mainly peg-shaped upper lateral incisors were also observed in about $4 \%$ of the sample. [5,6] Several genetic and syndromic conditions9-10 are known to the risk of hypodontia but congenitally missing teeth commonly are encountered in healthy apparently normal people $[3,5,6]$ Congenitally missing lateral incisors are a common dental problem that can be esthetically dealt in three different ways :

1. canine substitution, 2. Tooth supported restoration, and 3. implant supported restoration. Tooth auto transplantation (usually premolar) and removable partial dentures are other, less commonly applied treatment options. In the case of only one lateral incisor missing, an additional problem of symmetry between the right and left side usually exists and needs to be addressed.[1,2,4,7,8] 


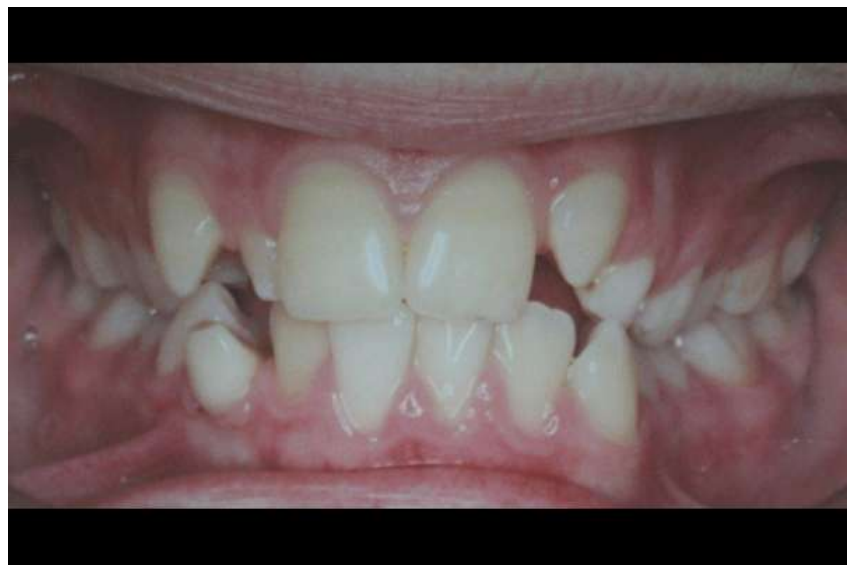

Fig.1; Clinical appearance at the age of 16 year

Peg shaped lateral incisors pose another aesthetic problem that is usually restored with as follows:

1. all ceramic crowns, 2. Porcelain veneers, and 3. direct or indirect composite veneers. Additional to the inadequate width and length of the peg shaped lateral, many times there is also a gingival aesthetic problem that can lead to a square looking restoration and too much gingival tissue display if not properly treatment planned with either orthodontic intrusion or gingivoplasty/ gingivectomy before the restoration is fabricated.[10,11,12,13]

In this article, a case is reported of a young patient with one congenitally missing and one peg shaped lateral incisor. The patient was treated with a combination of orthodontics, periodontal surgery and aesthetic restorative dentistry interventions.

\section{Case Report}

A-16year old male with a congenitally missing lateral incisor (22) with retained deciduous predecessor (62) and a peg shaped lateral incisor (12). (Figures 1,2)

The patient was diagnosed 8 years earlier and followed up annually during subsequent years for monitoring of development as well as prophylactic measures to maintain and improve his oral hygiene. At the age of 16 he was referred to the orthodontist. The patient exhibited a class I malocclusion with a class III skeletal pattern, an anterior open bite tendency, a slight midline shift and crowding of both dental arches. The 22 is congenitally absent with tooth 23 in the 22 position and the 12 is a peg-shaped lateral incisor.

The chief complaint of the patient was spaces between the teeth and speciically the missing upper left lateral incisor tooth, the irregularly shaped upper right lateral incisor, and the diastema between teeth \#11 and 21. Also, she was concerned about asymmetries in her smile and misalignment of her teeth. Finally, the patient stated she would like to have a brighter smile.
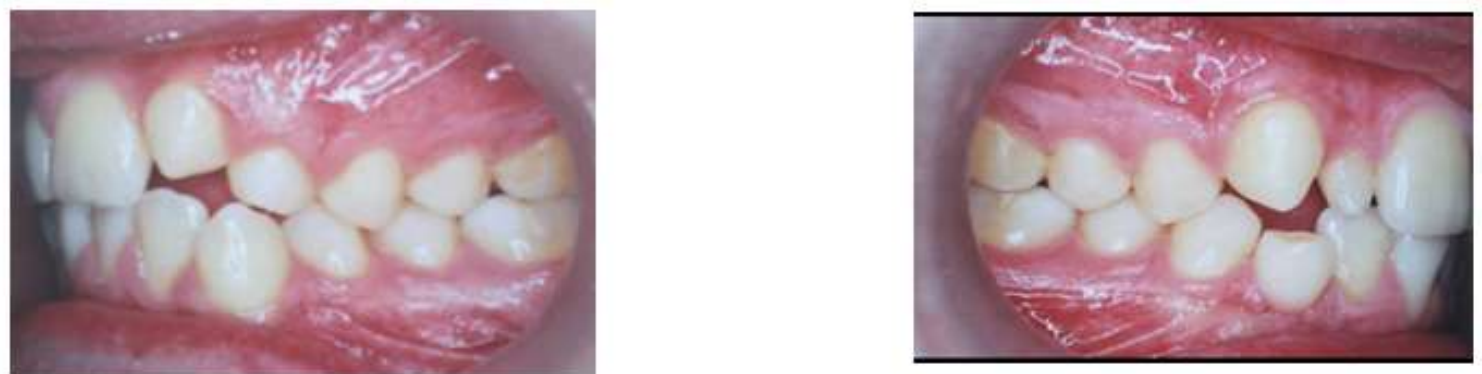

Fig.2; Peg shaped 12., The 23 has erupted into the 22 position and the 63 is retained.

The aesthetic evaluation of her smile resulted in the following issues that would need to be addressed in the treatment plan:

1. peg shaped lateral incisor \#12,

2. congenitally missing lateral incisor \#22 with diastema between\#11 and 21,

3. dental midline transmitted to the right by $4 \mathrm{~mm}$,

4. asymmetry between the left and right side, especially in the space between 11-13 and 21-23,

5. gummy smile, especially on the area of \#12 and the missing tooth \#22, and

6. the gingival zenith was asymmetrical between \#11 and 21 
Photographs and alginate impressions were taken in the exam appointment to fabricate study models. Then the team of aesthetic/restorative dentist, orthodontist and periodontist treatment planned the case. The recommended treatment plan was accepted by the patient in favor of the alternative treatment plans.

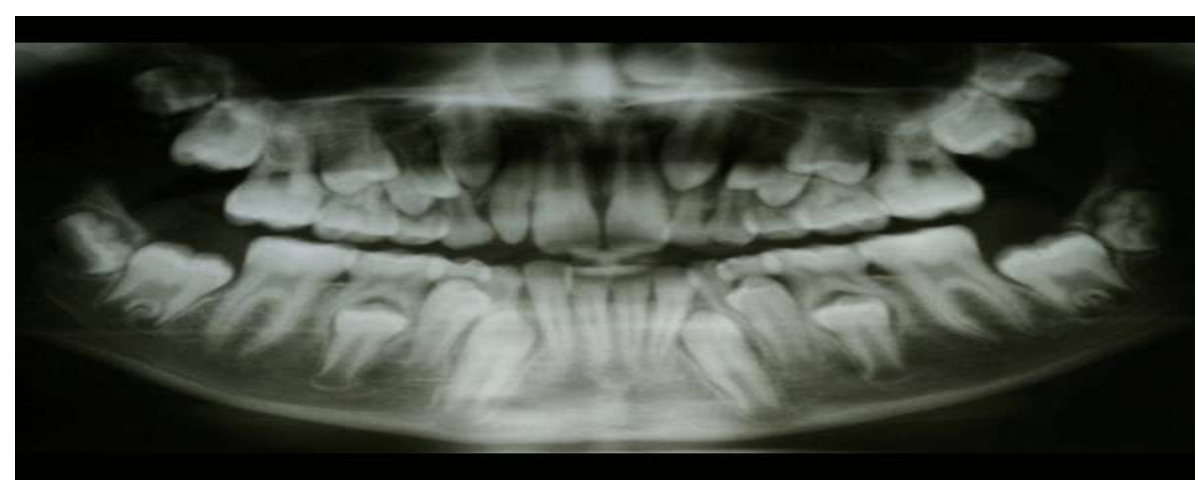

Fig.3; Panoramic radiograph at the age of 14 years

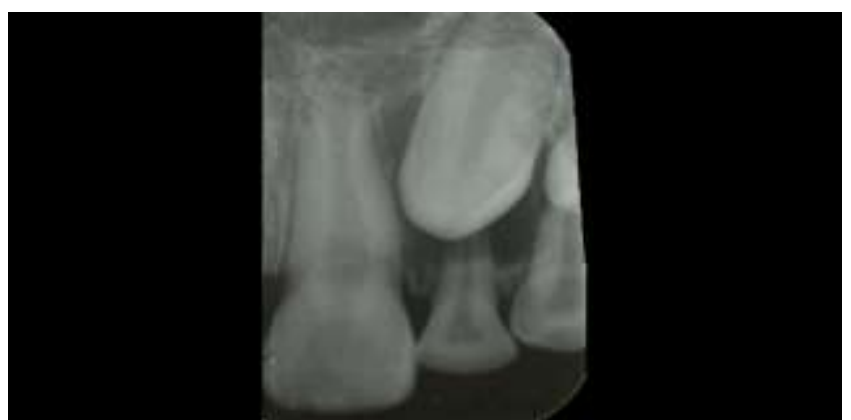

Fig.4; Periapical radiograph showing the 23 in the position of the congenitally absent 22 .

\section{-Orthodontic phase}

The orthodontic treatment goals were as follows: 1. intrude \#11 to align the incisal edges of the centrals, 2. equalize the spaces between \#11-13 and \#21-23, 3. transfer the dental midline to the left, and 4. correct misalignments and minor rotations in different areas. Some composite resin was bonded on the facial surface of tooth \#12 to facilitate bracket placement. The composite was white in shade to make it easier to distinguish and completely remove it after the orthodontics was completed. After treatment, the goals set were accomplished (Figures 7-9).

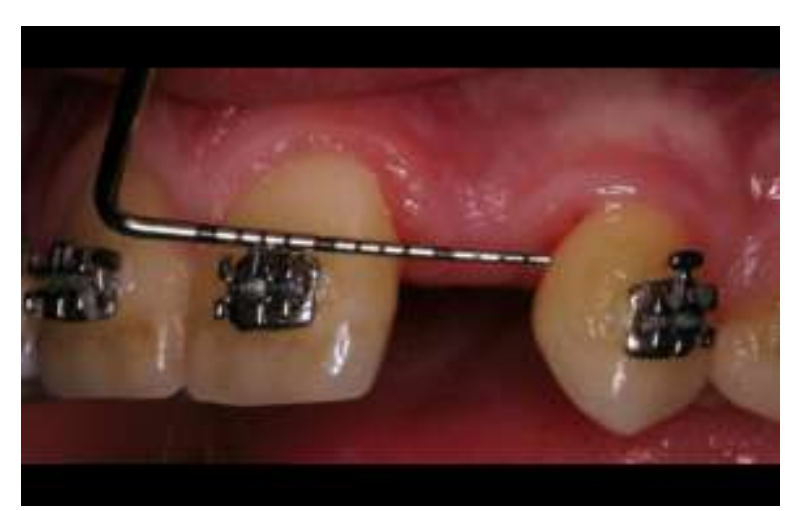

Fig.5; Pre-operative viev showing $6 \mathrm{~mm}$ intedental space.

\section{-Surgical phase A}

As stated previously, the dental team decided to align the incisal edges of \#11 and 21 and not intrude further \#11 to align the gingival zeniths. This decision was based on the fact thatmthe teeth showed no signs of wear, in which case the worn tooth would be intruded more to be back in its original prewear position and then would be treated restoratively. The goals of the periodontal surgery were 1. align the gingival zeniths of teeth $\# 11$ and 21,2. Gingivectomy with osseous reduction on \#12 to reduce as much as possible the gingival display without compromising the long term prognosis of the tooth due to loss of periodontal support, 3 . Gingivectomy in mostly all the upper teeth to bring the gingival display to a more pleasing appearance.

DOI: $10.9790 / 0853-1510018391 \quad$ www.iosrjournals.org $\quad 85 \mid$ Page




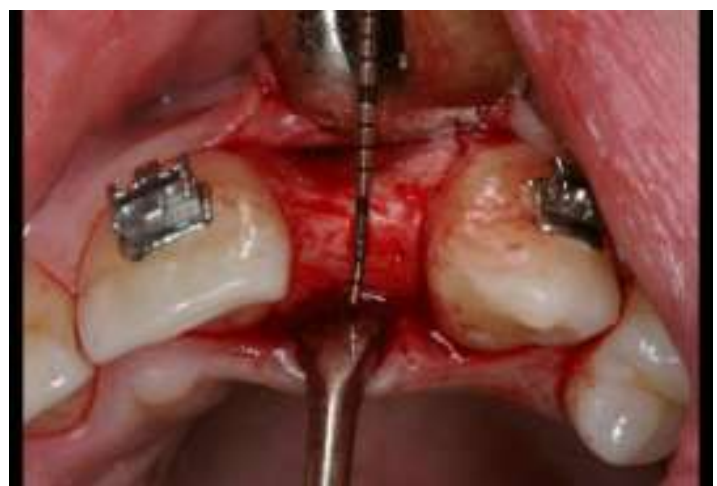

Fig.6; The bucco-palatal dimension is $4 \mathrm{~mm}$

After surgery, a healing period of 8 weeks was recommended by the periodontist before the restorative procedures start. The option of a single implant placement for the missing lateral incisor \#22 was rejected before surgery, as an additional bone grafting procedure would be required and this was not accepted by the patient.

\section{-Surgical phase B}

A more recent option for treating congenitally missing lateral incisors, and one that currently is recommended often, is the single-tooth implant. Over the past several years, the predictability and longterm success rates of implants have made them an obvious restorative choice, especially when teeth adjacent to the space are healthy, of normal size and shape, and unrestored. Furthermore, placement of an implant may provide a functional stimulus to help preserve bone and prevent resorption. However, when choosing the single-tooth implant as a restorative option, several factors must be taken into account such as growth considerations, space requirements, and site development.[14,16,16,17,18]

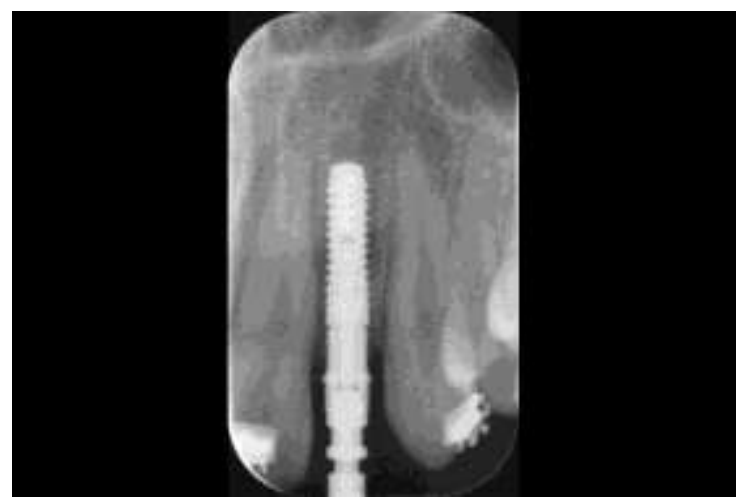

Fig.7; Final implant position.

Because an implant acts essentially like an ankylosed tooth, any vertical alveolar growth and eruption of teeth would cause a discrepancy between the gingival margin of the natural tooth and the implant.Therefore, implant placement should occur only after growth has been completed, and it has been suggested that neither chronological age nor hand-wrist radiographs are reliable enough to make that determination. Instead it would be best to compare superimposed cephalometric radiographs taken at 1-year intervals until no growth changes are detected $[16,17,19,20,21]$.

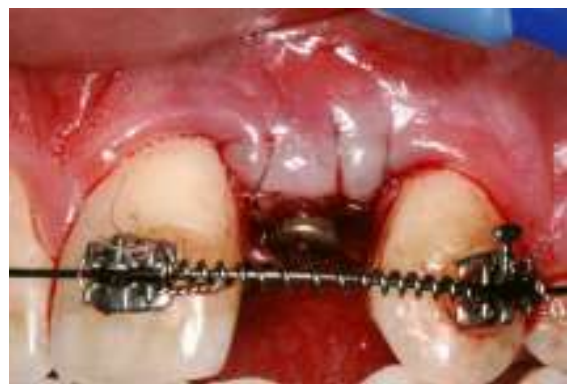

Fig.8; Replacement of the arch wire to prevent tooth movement during the integration period. 
Also, the amount of space between the roots is critical to successful implant placement, and orthodontic intervention usually is necessary to achieve not only the amount of interradicular space needed, but also the proper rootarigulation.Because orthodonti treatment usually occurs at an early age, several years of maintenance therapy may be required until the appropriate age for implant placement. It is also important to maintain proper spacing for ideal tooth proportions of the final restoration In addition to the tooth width requirements for mesiodistal spacing, the alveolar width in a buccolingual direction must be adequate for implant placement. Often an additional surgical appointment is necessary to graft or augment the alveolar ridge before an implant can be placed. It has been suggested in the literature that by allowing or guiding the eruption of the canines into the lateral position and orthodontically moving them to their natural position, the necessary amount of buccolingual alveolar thickness for implant placement can be achieved naturally, without the need to perform any ridge augmentation .[22.23,24,25,26]

When agenesis of maxillary lateral incisors is diagnosed in a young patient, usually primary maxillary lateral incisors are retained. In such cases, it may be necessary to selectively extract the primary lateral incisors to encourage the permanent canine to erupt mesially, adjacent to the central incisor. [27,28,29]

The canine will influence the thickness of the edentulous alveolar ridge due to its large buccolingual width; otherwise the osseous ridge will not fully develop due to the absence of the lateral incisor. $[2,4,18,30]$

As the canine is moved distally to open space for the lateral incisor implant and crown, the root movement creates an increased and adequate alveolar ridge which allows proper implant placement. However, the time of implant placement should be relative close to the orthodontic treatment. This procedure is called "Implant site development". If inadequate alveolar ridge is present, ridge augmentation may be necessary using bone grafts [1-8,19].

Adequate implant space: The amount of space needed for the implant and crown is generally determined by the contralateral lateral incisor. However, if both lateral incisors are missing or the contralateral one is peg-shaped, the amount of space should be determined by one of the methods below:

a.The golden proportion or a recurrent esthetic proportion

b. The Bolton analysis

c.A diagnostic wax-up

d. Mean values

The small size of the maxillary lateral from 5,5-8,0 $\mathrm{mm}$ requires careful planning for an implant to be placed. Is important that orthodontic movement has distanced not only thecrowns, but the roots of the adjacent teeth too. Generally, the adequate coronal space should be no less than $6,3 \mathrm{~mm}$ whereas the interradicular space no less than $5.7 \mathrm{~mm}$. «At least, $1,5 \mathrm{~mm}$ between of the implant and adjacent roots is desirable as it is cited that narrower distances between them are more likely to show a reduction in bone height over time. In addition, fixed retention is suggested rather than removable appliances to prevent relapse. crowns, but the roots of the adjacent teeth too $[2,9,10,11,19,20]$. Generally, the adequate coronal space should be no less than $6,3 \mathrm{~mm}$ whereas the interradicular space no less than $5.7 \mathrm{~mm}$. «At least, $1,5 \mathrm{~mm}$ between of the implant and adjacent roots is desirable as it is cited that narrower distances between them are more likely to show a reduction in bone height over time. In addition, fixed retention is suggested rather than removable appliances to prevent relapse. $[21,24,26,28,29]$

Generally, implants must not be placed until the patients have completed their facial growth and the majority of their tooth eruption $[2,6,8,11,30,31]$. As the face grows and the mandibular rami lengthen, teeth must erupt to remain in occlusion. However, the implant behaves like an ankylosed tooth and will not follow the changes of the alveolar processes due to the eruption of adjacent teeth. This may result in clinical infra occlusion of the implant supported crown and cause a discrepancy in the occlusalplane and between the gingival margins of the implant and the adjacent natural teeth. Thus, evaluation of the completion of facial growth by cephalometric radiographs must be done and subsequently, the patient should be informed for the optimal time of implant placement. However, even mature adults can exhibit major vertical steps after anterior restorations with implants to the same extend as adolescents .[5,8,12,16,18,22,34,26,27,29,31]

\section{-Aesthetic/restorative phase \#22}

Six weeks after surgery the patient returned for the restorative phase of treatment. The healing abutment on the implant was then modified to create a better emergence profile $(1,2, \%)$. This was achieved with air abrasion of the healing abutment, application of metal primer, bonding agent and flowable composite.

The desired effect was achieved in that the soft tissue moved in a bucco-apical direction creating a more labial emergence profile. A harmonious gingival contour with the adjacent teeth was established. It was suggested from the outset that a crown lengthening procedure on the peg shaped lateral would create a longer crown length and a more symmetrical gingival contour in relation to the contra-lateral incisor.[4,7,8,11] The patient decided to keep treatment simple and avoid further surgery and cost. [2] 
An open tray NC impression coping was connected to the implant and verified radiographically. The 22 was minimally prepared for a full coverage veneer. A polyether impression compound was used to take the final impression, taking great care to record the soft tissue emergence profile. A customised final abutment was cast accordingly and torqued to $35 \mathrm{Ncm}$. The porcelain fused to metal crown was cemented with Tempbond. The Emax full coverage veneer was luted with transparent Rely-X veneer cement, and the upper Hawley retainer adjusted to fit.

\section{-Aesthetic/restorative phase\#12}

The first step in any reconstruction is to determine shade and opacity using mock-ups of composite resin. This is a trial and error procedure where various materials are positioned and spot cured. Microfil composite has good handling properties and an excellent lustre but is very translucent. The surrounding teeth were rather opaque and it was decided to instead use predominantly nano hybrids with opacity in the mid range.

A natural tooth not only displays gradations of shade but also depth, particularly in young people where the enamel is translucent. Artists achieve subtlety and a sense of three dimensions by painting in increments, with undercoats effectively casting shadows through subsequent surface layers. In this case, a similar approach was to be used with the tooth built up in stages.[31]

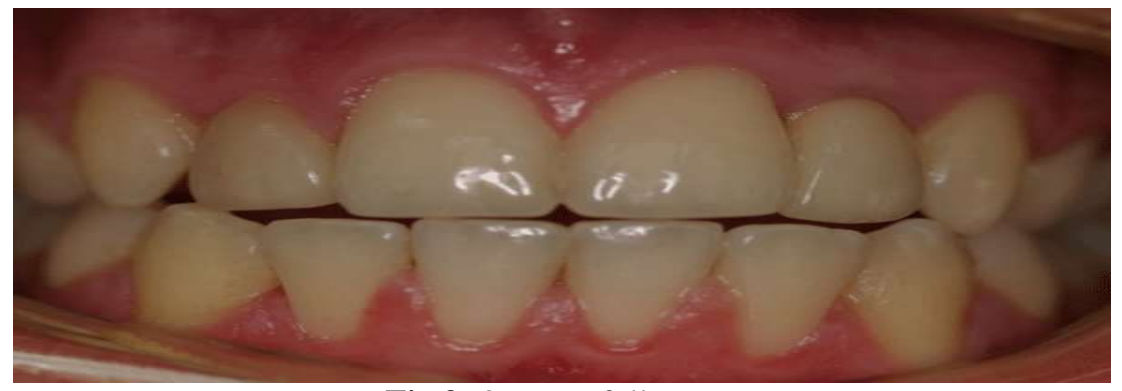

Fig.9; 3-years follow-up

A small, thin amount of A2 was placed and cured on the labial in the cervical third. A1 was applied to the mid portion and, before curing, tiny indentations created with the tip of a probe. Small flecks of opaque white tint were then added to the hollows and onto the incisal enamel to mimic the fluorosis spots seen on the neighbouring teeth.

To aid the flow of the next layer and prevent voids, this initial build up was smeared with a small amount of resin adhesive, blown with air and left uncured. Using plastic matrices angled into the cervical margins, B1 was then placed mesially and distally and manipulated onto the labial, over the earlier material, and onto the incisal.

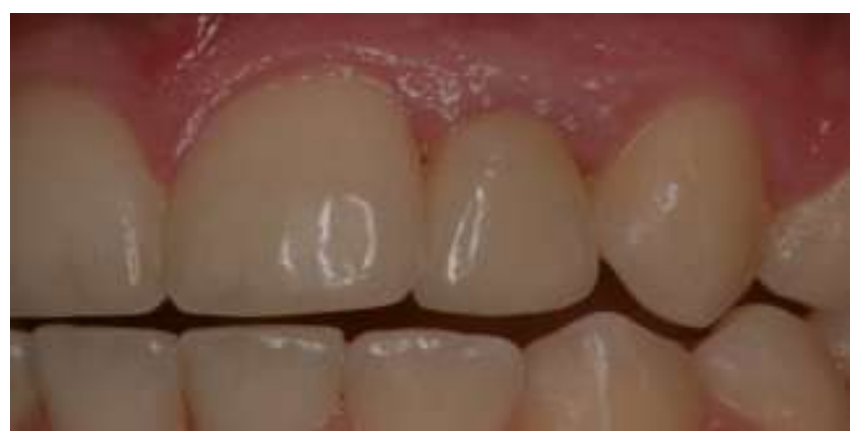

Fig.10; Implant 5-years follow-up.

It is difficult to ensure viscous composite always extends fully into crevices between teeth but, if the plastic strip is pulled palatally as the material is applied, friction will drag it interproximally. Invariably some irregularities are found on the palatal margins.

These were corrected with flowable composite, cured under a wash of glycerine to prevent any air inhibited layer. The build up was then shaped with disks, FG diamond burs and abrasive strips. Football shaped tungsten carbide burs were used to create undulations and scatter light. Long straight multi-fluted burs and rubber cones were then used to polish the surface.

The importance of good plaque control around the margins was emphasised, together with the need for regular review and maintenance. 


\section{Discussion}

Maxillary lateral incisors are often missing, misshaped, or small. Particular shapes that recur have been identified (eg, peg and barrel), and systems havebeen established so that dental anthropologists can nominally can nominally categorize misshaped or anomalous teeth.[8,9] Dental anomalies can result from many factors both genetic and environment. Although defects in certain genes are the most influential etiological events in the prenatal period. Post natal periods have also been blamed for anomalies in tooth dimensions, position, and number.[10] Peg shaped laterals are dental anomalies that are likely to be connected to defects in certain genes. There is probably a strong component of heredity, and peg shaped lateral incisors have been linked genetically with tooth agencies.[1,4,6,8,9]

The most compelling evidence for the genetic etiology of tooth agenesis has been provided by the identification of gene defects associated with different types of tooth agenesis. Dominant defects in Msx1, Pax9 and AXIN2 have been described in families with isolated severe tooth agenesis. However, in association with defects in Msx1, nail dysplasia and some patients with oral clefts have each been described in single families. In addition to causing severe tooth agenesis phenotype, a defect in AXIN2 also predisposed to colorectal cancer. Recently, two defects that affected only dentition were also described in Eda. All these gene defects cause severe types of agenesis. However, evidence for association of specific intragenic polymorphisms to tooth agenesis, apparently consisting mostly of common types of tooth agenesis, has been presented for Msx1, Pax9, AXIN2, TGFa, IRF6 and FGFR1.[3]

Tooth agenesis is associated with other anomalies of teeth. Tooth agenesis is prone to cause abnormal occlusion, the severity of which is dependent on the amount of missing teeth. Agenesis may also affect craniofacial development, and especially maxillary retrognathism and reduced anterior facial height have been reported [11]. A reduction in jaw size has also been described. However, as these may be considered secondary to agenesis, they will not be discussed further here. Several studies have addressed associations of agenesis and other dental anomalies [3,15]. Agenesis is often associated with reduction in tooth dimensions and morphology, delays of development, root anomalies, abnormal positions and also enamel hypoplasia [3,9,15] . However, these studies have not, with some exceptions, considered different subtypes of agenesis. While the following refers to studies concerning isolated tooth agenesis, these anomalies are rather often present in syndromic forms of tooth agenesis . [3,9]

The Msx1 and Msx2 genes have arisen by two successive gene duplication events acquiring their expression properties. During mid gestation, Msx1 and Msx2 expressions occur at almost all sites of epithelialmesenchymal tissue interactions. At E11.5, Msx1 is coexpressed with Msx2 in the dental mesenchyme .[3] However, the Msx1 is expressed quite broadly (in high levels) in the mesenchyme and the Msx2 expression is restricted to the mesenchyme around the tooth-forming regions. Msx1 is also strongly expressed in the developing molar and incisor tooth germs in a distal-to-proximal gradient in mesenchymeof the mandibular and maxillary processes. The expression of Msx1 in the dental mesenchymal tissue remains high throughout the cap stage, being down regulated at the early bell stage. [3] The Msx2 gene is expressed at early cap stage in the enamel knot, in the internal enamel epithelium and the dental papilla mesenchyme. With odontoblast differentiation, Msx2 becomes strongly expressed in the odontoblastic cells8. Mice deficient for Msx1 exhibit an arrest in molar tooth development at E13.5 bud stage, while mice deficient for Msx2 exhibit defects in cuspal morphogenesis, root formation and enamel organ differentiation.[3]

PAX9 is a member of a gene family encoding transcription factors that play a key role during embryogenesis. Proteins encoded by PAX genes share a unique 128-amino acid long DNA-binding paired domain $[3,8,9]$. PAX9 gene products function primarily by binding the enhancer DNA sequences and by modifying transcriptional activity of downstream genes [3,9]. To date, 11 distinct disease-causing mutations in the PAX9 gene (59 patients in 15 families) have been identified in humans, most of which are located in the paired box domain of PAX9. In contrast to MSX1, both missense and frame-shift mutations in PAX9 have been associated with hypodontia. Of the seven identified missense mutations, one is a premature termination mutation , and the remaining six are all residue substitution mutations. [3] Of these substitution mutations, only five generate a substitution in the protein , with one believed to prevent PAX9 expression. Three frame-shift mutations have been identified, two of which are caused by the insertion of a single nucleotide and the other by the deletion of eight nucleotides with the insertion of 288 foreign nucleotides [3,21]. Some of the unique reported mutations, like frame-shift, insertion, missense, nonsense and deletions of entire PAX9 gene.[22] These mutations were identified in the DNA-binding paired domain of PAX9 gene, resulting in a disturbed regulatory process occurring for tooth formation.[3,15] Missense mutations in PAX9 gene at amino acid position Gly6Arg and Ser43Lys were detected in two Chinese patients with non-syndromic tooth agenesis. $[3,23]$ Patients and their family members affected with oligodontia and other dental anomalies were carrying transition and nonsense mutation at $\mathrm{C} 175 \mathrm{~T}$, resulting in an altered arginine 59 Stop codon, thus leading to premature chain termination in PAX9 gene.[2,3] 
Peg shaped lateral incisors occur in approximately $2 \%-5 \%$ of the general population, and women show a slightly higher frequency than men. Usually they are found equally on the right and left, uni- or bilaterally, however some studies have shown their bilateral occurrence slightly higher than the unilateral occurrence. When peg shaped laterals erupt in the mouth, esthetically it can be a disappointment to the patient that their teeth are not perfect or too small in comparison to the rest of the anterior teeth. [1,2]Diagnosis is usually made clinically when peg lateral erupts. Treatment could be the combination of orthodontic treatment first to align the teeth in the arch, direct composite bonding onto peg laterals, indirect composite placement, bonded crowns, porcelain bonded to metal crowns, crown lengthening surgery to get better gingival heights then direct bonding, extractions, and implant placement.

The present case report demonstrates the interrelationship between various specialties of dentistry to achieve harmonious results in the form of ideal symmetry, proportion, and esthetics[2,5,8,9].

Peg lateral is usually associated with other dental anomalies like tooth agencies maxillary canine first premolar transposition4, palatal displacement of one or both maxillary canine teeth6, buccally displace Canine , and mandibular lateral incisor-canine transposition. In cases with concommitment dental anomalies, the prevalence suffers significant increase from normal prevalence.

The prevalence of hypodontia and congenital malformation in permanent teeth of Saudi Arabian male children was present in about 2.6-4\%; most frequently affected was the mandibular second premolars, maxillary laterals, and maxillary second premolars. Tooth malformations, mainly peg-shaped upper lateral incisors were also observed in about $4 \%$ of the sample.[32]

A retrospective study to assess the prevalence and distribution of hypodontia in the permanent dentition, excluding the third molars, in a sample of Japanese orthodontic patients found the prevalence of hypodontia to be $8.5 \%$ ( $7.5 \%$ for boys, $9.3 \%$ for girls) with no statistically significant difference between the sexes.[33,34]

Peg-shaped and reduced size upper lateral incisors were observed in $2.3 \%$ and $2.9 \%$ of the sample respectively, in a Jordanian sample.[35] In a retrospective study in Turkey the prevalence of tooth agenesis was $6.2 \%$, missing as a result of the congenital condition. Tooth agenesis was found more frequently in females than in males.[36] In another study in Mexico it has been seen that occurrence of congenitally missing teeth in the permanent dentition of a Mexican population most often affects third molars, followed by maxillary lateral incisors and then mandibular secondpremolars. $[37,38]$

Early diagnosis and the effective clinical management of hypodontia are important because the condition can lead to esthetic, physiologic, and functional problems .[39] In order to maintain various treatment options in the future, some authors defended that early orthodontic intervention may eliminate some of the periodontal and restorative problems that could arise in these patients as adults .[40] For example, as a result of extracting the maxillary primary lateral incisor and guiding the eruption of the permanent canine into the lateral incisor space, an excellent implant site can be developed in the mixed dentition. In contrast, other authors stated that deciduous teeth should be retained if present, in order to preserve alveolar bone after tooth extraction, since alveolar bone resorbs rapidly after tooth extraction.[40]

\section{Conclusions}

A multidisciplinary approach in treatment planning and performance, as well as the use of contemporary restorative materials and techniques allow for a conservative, yet very aesthetic final result.

\section{References}

[1]. Abusalih A. , Ismail H , Abdulgani A. , Chlorokostas G ., Abu- Hussein M . ; Interdisciplinary Management of Congenitally Agenesis Maxillary Lateral Incisors: Orthodontic/Prosthodontic Perspectives, Journal of Dental and Medical Sciences2016 , 15 ( 1) , 90-99 DOI: 10.9790/0853-15189099

[2]. Abu-Hussein M., Watted N., Abdulgani A., BorbélyB.Modern Treatment for Congenitally Missing Teeth : A MultidisciplinaryApproach; INTERNATIONAL JOURNAL OF MAXILLOFACIAL RESEARCH2015, 1(2);179-190

[3]. Abu-Hussein M., Watted N., Yehia M., Proff P., Iraqi F. ; Clinical Genetic Basis of Tooth Agenesis, Journal of Dental and Medical Sciences2015 ,14(12),68-77 DOI: 10.9790/0853-141236877

[4]. Abu-Hussein M, Watted N, Hegedủs V, Péter B . Congenitally Missing Upper Laterals. Clinical Considerations: Orthodontic Space Closure.Journal of Dental and Oral Health 2015, 1 ( 3),14,1-6

[5]. Muhamad Abu-Hussein, Nezar Watted, Abdulgani Azzaldeen, Mohammad Yehia, Obaida Awadi, Yosef Abu-Hussein. Prevalence of Missing Lateral Incisor Agenesis in an Orthodontic Arabs Population in Israel (Arab48). International Journal of Public Health Research. Vol. 3, No. 3, 2015, pp. 101-107.

[6]. Muhamad Abu-Hussein, Nezar Watted, Ali Watted, Yosef Abu-Hussein, Mohammad Yehia, Obaida Awadi, Abdulgani Azzaldeen. Prevalence of Tooth Agenesis in Orthodontic Patients at Arab Population in Israel. International Journal of Public Health Research.Vol. 3, No. 3, 2015, pp. 77-82.

[7]. Muhamad Abu-Hussein1, Nezar Watted2, Azzaldeen Abdulgani3An Interdisciplinary Approach for Improved Esthetic Results in the Anterior Maxilla DiastemaJournal of Dental and Medical SciencesVolume 14, Issue 12 Ver. VIII (Dec. 2015), PP 96-101DOI: $10.9790 / 0853-1412896101$ 
Multidisciplinary Aesthetic Dental Treatment; Peg lateral with Congenitally Maxillary lateral...

[8]. Abu-Hussein M., Abdulgani A., Watted N .Zahalka M. ; Congenitally Missing Lateral Incisor with Orthodontics, Bone Grafting and Single-Tooth Implant: A Case Report. Journal of Dental and Medical Sciences2015 , 14(4),124-130 DOI: 10.9790/08531446124130

[9]. Abdulgani A.,. Kontoes N., Chlorokostas G.,Abu-Hussein M .;Interdisciplinary Management Of Maxillary Lateral Incisors Agenesis With Mini Implant Prostheses: A Case Report; Journal of Dental and Medical Sciences2015 ,14 (12) , 36-42 DOI: $10.9790 / 0853-141283642$

[10]. Nezar Watted ,Abdulgani Azzaldeen ,Muhamad Abu-Hussein ;AESTHETIC REPLACEMENT OF CONGENITALLY MISSING TOOTH USING FIBER-REINFORCED COMPOSITE (FRC) Int J Dent Health Sci 2014; 1(4): 644

[11]. Muhamad Abu-Hussein , Nezar Watted ;Maxillary Midline Diastema - Aetiology And OrthodonticTreatment- Clinical ReviewJournal of Dental and Medical Sciences2016, 15, 6, 116-130DOI: 10.9790/0853-150602116130

[12]. Abu-Hussein Muhamad*, Dr. Abdulgani Azzaldeen , Dr.Abdulgani Mai;Esthetics of Class IV Restorations with Composite ResinsJournal of Dental and Medical Sciences 2016,15, 1 ,61-66DOI: 10.9790/0853-15126166

[13]. Muhamad Abu-Hussein1, Nezar Watted2, Azzaldeen Abdulgani3An Interdisciplinary Approach for Improved Esthetic Results in the Anterior Maxilla DiastemaJournal of Dental and Medical Sciences2015, 14, 12 . 96-101.DOI: 10.9790/0853-1412896101

[14]. Abu-Hussein M , Chlorokostas G, Watted N, Abdulgani A, Jabareen A, ,Pre-Prosthetic Orthodontic Implant for Management of Congenitally Unerupted Lateral Incisors - A Case Report Journal of Dental and Medical Sciences2016, 2 .Vol 15 (2 ), 99-104 DOI: $10.9790 / 0853-152899104$

[15]. Abu-Hussein M., Watted N., Abdulgani A., Kontoes N .; Prosthodontic-Orthodontic Treatment Plan with Two- UnitCantilevered Resin-Bonded Fixed Partial Denture, IOSRJDMS 2015,14(12) , 131-136 DOI: 10.9790/0853-14124131136

[16]. Abdulgani A.,. Kontoes N., Chlorokostas G.,Abu-Hussein M .;Interdisciplinary Management Of Maxillary Lateral Incisors Agenesis With Mini Implant Prostheses: A Case Report; IOSR-JDMS2015 ,14 (12), 36-42 DOI: 10.9790/0853-141283642

[17]. Abu-Hussein M , Chlorokostas G, Watted N, Abdulgani A, Jabareen A., Pre-Prosthetic Orthodontic Implant for Management of Congenitally Unerupted Lateral Incisors - A Case Report Journal of Dental and Medical Sciences2016, Vol 15 (2 ), 99- 104 DOI: $10.9790 / 0853-152899104$

[18]. Muhamad AH, Azzaldeen A, Nezar W, Mohammed Z. ; Esthetic Evaluation of Implants Placed after Orthodontic Treatment in Patients with Congenitally Missing Lateral Incisors. J Adv Med Dent Scie Res2015 ;3(3):110-118

[19]. Abdulgani M. , Abdulgani Az ., Abu-Hussein M .; Two Treatment Approaches for Missing Maxillary Lateral Incisors: A Case Journal of Dental and Medical Sciences 2016,Volume 15, Issue $7,78-85$ DOI: 10.9790/0853-150787885

[20]. Muhamad Abu-Hussein et al, Congenitally Missing Lateral Incisors; Orthodontic, Restorative, and Implant Approaches. Int J Dent2016, 2:2, 71-84

[21]. Abu-Hussein M, Watted N, Hegedüs V, Péter B . Congenitally Missing Upper Laterals. Clinical Considerations: Orthodontic Space Closure.Journal of Dental and Oral Health 2015, 1 ( 3),14,1-6

[22]. Muhamad AH ; Managing congenitally missing lateral incisors with single tooth implants, Dent Oral Craniofac Res2016,2(4): 318324. doi:10.15761/DOCR.1000169

[23]. Abu-Hussein M, Watted N, Shamir D ) A Retrospective Study of the AL Technology Implant System used for Single-Tooth Replacement. Int J Oral Craniofac Sci 2016,2(1): 039-046. DOI: 10.17352/2455-4634.000017

[24]. Nezar Watted , Abdulgani Azzaldeen, Ghannam Nidal, Ali Watted ,Abu-Hussein Muhamad Congenitally Missing Bilateral Incisors with Single-Tooth Implants: Clinical CaseJournal of Dental and Medical Sciences 2016 . 15, 9; 84-90DOI: 10.9790/08531509058490

[25]. Abu-Hussein Muhamad,Watted Nezar ,Abdulgani, Azzaldeen;Esthetic Management of Congenitally Missing Lateral Incisors With Single Tooth Implants: A Case ReportJournal of Dental and Medical Sciences 2016, 15, 8, 69-75DOI: 10.9790/0853-1508096975

[26]. Muhamad AH, Azzaldeen A ;Autotransplantation of Tooth in Children with Mixed Dentition. Dentistry2012, 2:149.doi:10.4172/2161-1122.1000149]

[27]. Muhamad Abu-Hussein1, Nezar Watted 2, Azzaldeen Abdulgani;Gummy Smile and Optimization of Dentofacial EstheticsJournal of Dental and MedicaSciencel2015, 14, 4 ,24-28DOI: 10.9790/0853-14462428

[28]. Abu-Hussein M. , Watted N .,Abdulgani M ., Abdulgani Az; Tooth Autotransplantation; Clinical Concepts Journal of Dental and Medical Sciences2016, 15 (7) 105-113 DOI: 10.9790/0853-15078105113

[29]. Abdulgani M. , Abdulgani Az ., Abu-Hussein M .; Two Treatment Approaches for Missing Maxillary Lateral Incisors: A Case .Journal of Dental and Medical Science2016,s 15, 7 , 78-85 DOI: 10.9790/0853-150787885

[30]. Abu-Hussein M, , Abdulgani Azzaldeen; Intentional replantation of maxillary second molar; case report and 15-year follow-up. Journal of Dental and Medical Sciences. 2016, 15, 1, 67-73 DOI: 10.9790/0853-15126773

[31]. Abu-Hussein Muhamad, Watted Nezar, Abdulgani Azzaldeen. The Curve of Dental Arch in Normal Occlusion. Open Science Journal of Clinical Medicine2015. 3, 2, 47-54

[32]. Abd-AzizHM, Foda MY. Prevalence of peg-shaped maxillary lateral incisor in relation to tooth agenesis and malposition of the maxillary cuspids in a group of Egyptian population. Egypt Dent J 2004; 50: 545

[33]. Al-Emran S. Prevalence of hypodontia and developmental malformation of permanent teeth in Saudi Arabian schoolchildren. $\mathrm{Br} \mathrm{J}$ Orthod 1990;17(2):115-8.

[34]. Endo T, Ozoe R, Kubota M, Akiyama M, Shimooka S. A survey of hypodontia in Japanese orthodontic patients.AmJ OrthodDentofacialOrthop 2006;129(1):29-35

[35]. Peck S, Peck L, Kataja M. Prevalence of tooth agenesis and peg-shaped maxillary lateral incisor associated with palatally displaced canine anomaly. Am J Orthod Dento facial Orthop 1996;110(4):441-3.

[36]. Cantekin K, Dane A, Miloglu O, Kazanci F, Bayrakdar S, Celikoglu M. Prevalence and intra-oral distribution of agenesis of permanent teeth among Eastern Turkish children. Eur J Paediatr Dent 2012;13(1):53-6.

[37]. Vahid-Dastjerdi E, Borzabadi-Farahani A, Mahdian M, AminiN. Non-syndromic hypodontia in an Iranian orthodontic population.J Oral Sci 2010;52(3):455-61.

[38]. Al-Mulla AA, Mahdi TS, Hamid NH. Incidence of Hypodontia of Permanent Teeth. Tech Res J1990; 7: 69-80.

[39]. Tunc, E. S.; Bayrak, S. et al. . Dental development in children with mild-to-moderate hypodontia. American Journal of Orthodontics and Dentofacial Orthopedics,2011, 139(3): 334- 338.

[40]. Kokich, V. . Early Management of Congenitally Missing Teeth. Seminars in Orthodontics 2005,11: 146-151. 